\title{
Preliminares para un análisis métrico-acentual de la poesía de Teócrito*
}

\author{
Alejandro Abritta \\ Universidad de Buenos Aires - Conicet \\ alejandroabritta@gmail.com \\ ORCID iD: http://orcid.org/0000-0001-6121-8917
}

\section{Preliminaries for a metrical-accentual analysis of Theocritus' poetry}

El presente artículo busca dar los primeros pasos en un análisis de la distribución de acentos en el hexámetro de Teócrito, a partir de la idea de que los poetas antiguos realizaban esta distribución de manera deliberada, avanzada recientemente por diversos autores. Luego de una introducción y algunas consideraciones metodológicas, se estudian los tipos de acentos en el tercer pie de un conjunto de dieciocho de los Idilios (aquellos cuya autoría corresponde con toda probabilidad a Teócrito), luego los tipos de acentos con los que el poeta cierra sus versos y, finalmente, se realiza un análisis de la correlación entre el tipo de acento en el tercer pie y el sexto. El estudio se propone detectar diferencias y similitudes entre los distintos poemas y grupos de poemas y verificar si se corresponden con las encontradas en el análisis metricológico.

Palabras clave: Teócrito; Hexámetro; Acento; Idilios.
This paper attempts to take the first steps in an analysis of the distribution of accents in Theocritus' hexameter, following the hypothesis, proposed recently by several scholars, that ancient poets distributed accents in a deliberate manner. After an introduction and some methodological considerations, the author studies the types of accent in the third foot of a group of eighteen Idylls (those that can be safely attributed to Theocritus), then the types of accent with which the poet closes his verses and, finally, he conducts an analysis of the correlation between the type of accent in the third and in the sixth foot. The study attempts to detect similarities and differences between the different poems and groups of poems, and to verify if they correspond with those found in the metricological analysis.

Key words: Theocritus; Hexameter; Accent; Idylls.

Cómo citar este artículo / Citation: Abritta, Alejandro 2018: «Preliminares para un análisis métricoacentual de la poesía de Teócrito», Emerita 86 (1), pp. 1-25.

* El presente trabajo ha sido posible gracias a la contribución del proyecto UBACyT código 2002013010053BA, financiado por la Universidad de Buenos Aires, y el proyecto PIP 11220120100145CO, financiado por el Conicet. Ambos son dirigidos por el Dr. Daniel Alejandro Torres, a quien expreso mi agradecimiento por su cuidadosa revisión de una versión preliminar del texto.

Copyright: (C) 2018 CSIC. Este es un artículo de acceso abierto distribuido bajo los términos de la licencia de uso y distribución Creative Commons Reconocimiento 4.0 Internacional (CC BY 4.0). 


\section{INTRODUCCIÓN}

\section{Proyecto}

Hace cuarenta años Brioso Sánchez publicaba su esencial trabajo sobre la métrica externa de Teócrito, el análisis cuantitativo más completo que se ha realizado sobre ella ${ }^{1}$. Casi veinte años después, Marco Fantuzzi complementó este estudio con un análisis de la métrica interna del poeta helenístico ${ }^{2}$. Se puede afirmar que entre los dos artículos agotan el enfoque formal sobre el hexámetro de Teócrito desde el punto de vista metricológico.

Si hasta no hace mucho esto implicaba que el estudio cuantitativo de los Idilios estaba razonablemente completo, ciertos cambios en nuestra comprensión de los metros griegos sugieren que en realidad este no es el caso. A partir de los trabajos de Nagy 2000 y 2010, Lascoux 2003 y David 2006, he buscado demostrar que el acento tonal del griego antiguo, que los manuales de métrica sostienen regularmente que no cumplía ninguna función en la composición poética ${ }^{3}$, en realidad sí la tenía (cf. Abritta 2015). En otras palabras, que los diferentes tipos acentuales de palabras eran distribuidos en forma deliberada por los poetas respetando ciertas tendencias. Esta nueva perspectiva sobre la poesía antigua sugiere que los trabajos mencionados al comienzo deben ser complementados con uno que analice el metro de Teócrito desde el punto de vista acentual. Este estudio pretende dar los primeros pasos en esa dirección ${ }^{4}$.

${ }^{1}$ Brioso Sánchez 1976-77 (a partir de este punto, «Brioso Sánchez»). Sobre la oposición métrica externa / métrica interna, cf. O’Neill 1942, p. 106 n. 2.

${ }^{2}$ Fantuzzi 1995 (a partir de este punto, «Fantuzzi»). Fantuzzi revisa también brevemente la métrica externa (pero apoyándose en Brioso Sánchez) y el uso de correptio attica en los diferentes Idilios. Debe mencionarse también en esta línea el trabajo de Di Benedetto 1956.

${ }^{3} \mathrm{La}$ oración «el acento tonal griego no cumplía ninguna función en la poesía» aparece en alguna forma en la mayoría de los manuales de métrica. E.g. West 1982, p. 2; Sicking 1993, p. 64.

${ }^{4}$ El proyecto implica el axioma de que el descubrimiento del rol del acento en la poesía homérica es trasladable a la helenística en general o al menos a la de Teócrito. Para evitar limitar esta noción a la de un mero postulado, he realizado sobre mi muestra de Teócrito las pruebas que llevé a cabo sobre Homero (cf. Abritta 2015). Los resultados divergen en ciertos puntos, pero la distribución de formas ỏ łús y ßapús en el verso (cf. Abritta 2015, pp. 13-14 y 24-25 y abajo, sec. I.2) permite inferir que el poeta helenístico colocaba los acentos en forma deliberada. 


\section{Problemas metodológicos}

El proyecto presenta dos grandes problemas. El primero, que no contamos con una serie clara y simple de principios métrico-acentuales del hexámetro que sea posible contrastar entre los diferentes Idilios o entre los Idilios y otros poemas. He decidido que para solucionar este inconveniente la mejor estrategia es seleccionar algunos aspectos de la distribución de acentos y contrastarlos en los diferentes textos del corpus teocríteo. En otras palabras, compensar la ausencia de reglas con un estudio de la distribución efectiva de determinados tipos acentuales. Es, por supuesto, menos certero analizar la proporción de circunflejos en un determinado punto del hexámetro que la incidencia de diéresis bucólica, no porque un dato sea más concreto u objetivo que el otro ${ }^{5}$, sino porque existe una larga tradición ritmicológica que permite dar sentido a una incidencia mayor o menor de diéresis bucólica, pero nada equivalente que facilite la interpretación de un porcentaje mayor o menor de circunflejos. Si del aspecto descriptivo del presente trabajo puede esperarse con buenos motivos que sentará bases sólidas para un estudio amplio de la métrico-prosodia ${ }^{6}$ de Teócrito y quizás de la poesía helenística en general, del aspecto explicativo sólo pueden surgir especulaciones e hipótesis que deberán ser corroboradas en un trabajo más extenso sobre el hexámetro y los metros griegos.

El segundo problema importante para el proyecto que se encara es la clasificación de los distintos Idilios. Es bien sabido que esta colección incluye una variedad de, a falta de una palabra más precisa, géneros poéticos. Los autores que han realizado análisis formales de estos textos (métricos o lingüísticos) los han agrupado sobre la base de determinados rasgos. Gow 1952, p. lxxii, es el ejemplo clásico de clasificación por dialectos: el editor identifica cinco grupos que se distinguen por un uso mayor o menor de dórico, épico, jónico y eólico, además de por su carácter genuino. Di Benedetto 1956 presenta el primer inconveniente de esta clasificación: en el grupo que Gow consideraba constituido por «poemas genuinos en dórico» hay en realidad un subconjunto constituido por poemas en dórico más bien puro y poemas en

\footnotetext{
${ }^{5}$ De hecho, si uno de esos dos datos fuera más concreto u objetivo, sería el del porcentaje de circunflejos, dada la complejidad teórica de la noción de cesura.

${ }^{6}$ Utilizo «métrico-prosodia» para referirme al aspecto del análisis formal del verso que se ocupa de la distribución de acentos en él y su relación con los rasgos de métrica interna y métrica externa.
} 
dórico matizado con elementos del lenguaje épico. Pero incluso con esta corrección la cuestión permanece abierta y, como observa Hunter 1996, pp. 31-38, son muchos los problemas para una clasificación dialectal.

Una estrategia alternativa sería utilizar criterios temáticos, pero además de la volubilidad de semejante estrategia, no es posible acomodar todos los Idilios en un conjunto reducido de grupos bien definidos. Hunt 2009 argumenta por una división entre poemas bucólicos (Id. I-VII, X-XI) y poemas no-bucólicos, que puede resultar útil, pero deja de lado diferencias muy evidentes de tono entre, por ejemplo, el $I d$. I, el $I d$. II y el $I d$. VI. Si para un análisis narratológico o literario la ampliación de la categoría «bucólico» es interesante, parece adecuado afirmar que, por decirlo de algún modo, los diferentes estados de ánimo de los personajes que toman la voz en los Idilios podrían haber tenido correlatos formales que la agrupación temática mayor no permitiría detectar. Esto no es decir que estos correlatos efectivamente existieran, sino que ante la posibilidad de que existan no es posible apoyarse en una agrupación temática.

La clasificación métrica sería probablemente la más adecuada. Sin embargo, el contraste entre los trabajos de Brioso Sánchez y Fantuzzi sugiere que no está libre de inconvenientes, dada la diferencia entre los autores en la clasificación de los Id. X y XI. Por lo demás, agrupar los Idilios para su análisis pareciera implicar adoptar una determinación a priori sobre aquello que se busca concluir. De acuerdo con mis datos, por ejemplo, el Id. XIV tiene una incidencia de diéresis bucólica de $69,57 \%$, mientras que el Id. XV de tan sólo 45,64\%: el primero está más cerca de los poemas bucólicos ${ }^{7}$ que el segundo ${ }^{8}$. Sin embargo, Brioso Sánchez los considera como parte del mismo grupo y Fantuzzi (p. 247) afirma que están relacionados. Naturalmente, la incidencia de diéresis bucólica no es el único criterio utilizado por los

\footnotetext{
${ }^{7}$ A partir de este punto, usaré siempre «poemas bucólicos» para el conjunto de los $I d$. I y III-VII, que tienen una incidencia promedio de diéresis bucólica de $80,09 \%$, pero un rango que va de $74,52 \%$ en el $I d$. VII a $88,59 \%$ en el $I d$. V.

${ }^{8}$ Los datos sobre la diéresis bucólica han sido obtenidos aplicando un «indicador de cesura», que cuenta palabras métricas (es decir, grupos de palabra léxica y sus clíticos) terminados en la ubicación de la cesura. Es plausible que este indicador sobreestime (o subestime) el porcentaje efectivo de cesuras en un texto (dependiendo de la definición de cesura que uno prefiera; no todos los autores consideran que la diéresis bucólica sea una en sentido estricto, de hecho), pero es útil para contrastar diferentes textos entre sí. Aprovecho para notar también que la inconstancia de Teócrito no escapó a Brioso Sánchez, que la observa en p. 59, n. 80.
} 
autores o utilizable, pero el fenómeno sugiere que, incluso entre poemas que parece verosímil considerar dentro de un cierto grupo genérico, puede haber diferencias considerables a la hora de analizar los datos.

$\mathrm{Y}$ es en este punto donde los dos problemas mencionados se imbrican. Al analizar la métrica o el dialecto de los Idilios es relativamente sencillo identificar qué debe rastrearse. Sin embargo, no hay un criterio definido para saber qué rasgos métrico-prosódicos (si los hubiera) diferencian un poema de otro, y por eso analizar los textos en grupos pre-determinados implica el riesgo de perder algunas distinciones clave. Por supuesto, realizar un estudio pormenorizado tiene otros costos, en particular las dificultades que conlleva trabajar con un conjunto grande de textos. En este punto de la investigación, no obstante, he preferido acotar la cantidad de pruebas a agrupar los Idilios.

\section{Corpus seleccionado}

El corpus de análisis que se ha seleccionado es el mismo utilizado por Brioso Sánchez, compuesto por los poemas cuya autoría puede afirmarse con relativa seguridad que corresponde a Teócrito. Este grupo incluye los Idilios I-VII, X-XVIII, XXII y XXIV. En el $I d$. XV he distinguido (siguiendo a Fantuzzi) dos partes: la sección mímica del diálogo entre las siracusanas (Id. XVa) y el canto a Adonis incluido en el texto entre los versos 100-144 (Id. XVb). He suprimido del análisis los versos que Gow considera interpolados, excepto los vv. 86-87 del $I d$. XXIV para cuya exclusión los argumentos del editor me resultan insuficientes.

En el caso de los poemas con un estribillo, es decir, los Idilios I y II, se ha incorporado sólo una instancia de cada variante del estribillo en el análisis (tres versos en el $I d$. I, dos en el $I d$. II $)^{9}$. He seguido (parcialmente) en esto a Brioso Sánchez y Fantuzzi, pero sin convicción. Un estribillo no es un recurso compositivo despreciable: sirve (entre otras cosas) para marcar el tono de un poema. Excluirlo de un estudio cuantitativo, aunque puede justificarse sobre la base de una búsqueda de una cierta equidad en la compilación de datos, parece ignorar lo importante de esta función ${ }^{10}$.

9 Para la posibilidad de un tercer estribillo en el $I d$. II, que habría sido transmitido en el desplazado v. 61, cf. Cavallero 1998.

${ }^{10}$ De cualquier manera, he revisado los datos incluyendo los estribillos, y las conclusiones obtenidas no se modifican en absoluto (de hecho, todo lo contrario). 
Tanto los datos analizados como las herramientas utilizadas son las provistas en https://greekmps.wordpress.com. Las tablas desarrolladas para este trabajo se publicarán también online (en https:/empgriegos.wordpress.com/ datos-experimentales/preliminares-para-un-analisis), no sólo para garantizar la accesibilidad de los números sobre los que este estudio se basa, sino también para ofrecer una cantidad de información que no es posible incluir en los acotados límites de un artículo.

\section{Modelo acentual}

El modelo fonético del griego antiguo está hoy en un nivel de desarrollo considerable, aunque diversos problemas permanecen abiertos ${ }^{11}$. El punto de inflexión fundamental en la historia de este modelo está en los trabajos de Allen 1967 y 1973 (entre otros), donde se busca demostrar que el acento tonal griego consistía en un movimiento melódico constituido por un ascenso del tono seguido de un descenso posterior que podía realizarse en la misma sílaba del ascenso (circunflejo) o en la siguiente (agudo), donde ocupaba la mora vocálica de una sílaba breve o las dos moras de una larga. Devine y Stephens 1991 y 1994 complementan este estudio con un análisis de los textos con notación musical (que Allen también había tomado en cuenta) demostrando, entre otras aportaciones, que un contorno tonal puede completarse en un enclítico y que el descenso del tono no es sólo una nivelación con las sílabas no acentuadas (como propuso Allen), sino que de hecho llega a un nivel melódico más bajo que ellas.

Este último fenómeno coloca a la parte descendente del contorno acentual en una posición distinta a la que tendría si fuera nada más que una nivelación. El esfuerzo extra de bajar el tono destaca la sílaba portadora del descenso como una sílaba acentuada de pleno derecho. A partir de esta observación, David 2006 reinterpreta la clasificación antigua de palabras basada en los términos ßapús y ỏ zús sobre la base de la hipótesis de que una parte de la contonación constituía la prominencia acentual principal: cuando el descenso del tono ocupa una extensión mayor que el ascenso (en los casos en que ocupa las dos moras de una sílaba larga después de un acento agudo, como

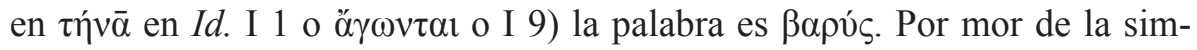

${ }^{11}$ Presento aquí solamente aquello del complejo campo de la fonética reconstruida del griego antiguo que sirve a los fines de este estudio. Probert 2003, pp. 97-124, presenta un resumen de una parte de la historia del área. 
plicidad, denominaré a este tipo de prominencia (sílaba larga post-agudo) «declive barítono» o "declive». Cuando ocupa una extensión igual o está

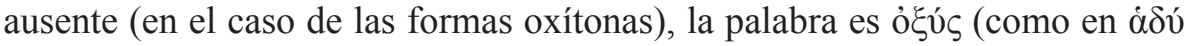

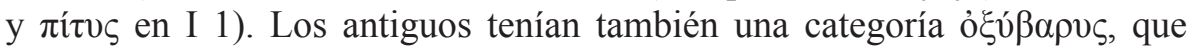
usualmente se identifica con el circunflejo ${ }^{12}$. Las formas perispómenas y properispómenas, sin embargo, son clasificadas por David dentro del grupo de las $\beta \alpha \rho u ́ s$, en la medida en que se considera que en ellas la prominencia tonal estaba sobre todo en el descenso del tono ${ }^{13}$.

Aparte de estos grupos regulares, hay una categoría de palabras para la cual existe evidencia de que tenían un comportamiento peculiar: las formas paroxítonas de final trocaico con penúltima sílaba con vocal breve cerrada

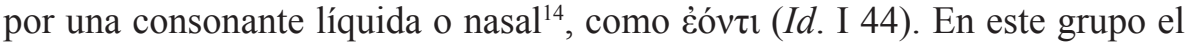
descenso del tono se completaba aparentemente en la consonante de la coda, dando a ésta un sonido similar al de un circunflejo. Deberá ser, por lo tanto, considerado por separado en los análisis que siguen.

Para otros aspectos más específicos del análisis he seguido las líneas que pueden hallarse en https://greekmps.wordpress.com/prosodical-bases. Como punto destacado debe notarse que en todos los casos he tomado las palabras en la forma que aparecen en el metro, no en su forma aislada. Así, las formas con elisión han sido contadas en su forma elidida (es decir que ó $\lambda \gamma \varepsilon$ ' en $I d$. I 19 es considerada como un disílabo trocaico paroxítono del grupo descripto en el párrafo anterior, no como el trisílabo proparoxítono que es la forma aislada - $\alpha \lambda \lambda \gamma \varepsilon \alpha-)$ y las palabras con sílaba final cerrada con las cantidades que tienen efectivamente en el metro ( $\sigma 0 \rho i ́ \sigma \delta \varepsilon \varsigma$ en $\sigma 0 \rho i ́ \sigma \delta \varepsilon \varsigma \cdot \mu \varepsilon \tau \grave{\alpha} . .$. en $I d$. I 3 no es un trisílabo de final trocaico, sino un trisílabo de final espondaico con prominencia $\beta \alpha \rho u ́ \varsigma$ en última). Asimismo, el análisis no se restringe a las palabras lingüísticas, sino que abarca también las palabras métricas. Un gru-

${ }^{12}$ Los testimonios son analizados por David 2006, pp. 53-68. Cf. también Sturtevant 1920, pp. 192-205.

${ }_{13}$ Existe una interpretación alternativa a la de David que conserva las categorías ỏ $u ́ c$ y ßapús como él las clasifica. Bapús (palabra que identifica un sonido fuerte y bajo) sería utilizado para formas donde había coincidencia de acento y prominencia rítmica (sobre el ritmo en griego cf. Devine y Stephens 1994, pp. 117-156 y 195-215) en la totalidad de una sílaba (el circunflejo y el descenso del tono post-agudo en una sílaba larga comparten invariablemente este rasgo). Las categorías ỏ zúc y ßapúc no serían así estrictamente tonales, sino que señalarían rasgos rítmico-tonales (algo que, por lo demás, está implicado en la propuesta de David).

${ }^{14}$ Cf. Abritta 2015, p. 14, con sus referencias. 


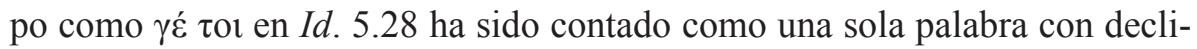
ve barítono en última (pero véase la n. 26).

\section{Interpretación de las marcas acentuales en la lectura de la poesía}

Incluso con un modelo fonético bien definido (incluso con uno mucho mejor definido que el que tenemos), el problema de cómo eran ejecutados los acentos en la lectura o el canto de la poesía persiste. Es una cuestión bien conocida en el análisis de la lírica ${ }^{15}$, pero no así en el caso de los textos considerados usualmente como «recitativos». Dada la complejidad de este tema, he determinado resolverlo para este trabajo en forma axiomática: se interpretará que los acentos eran ejecutados con su tono natural, quizás de manera algo más enfática o marcada que en el lenguaje cotidiano, pero sin modificaciones tonales significativas. Debe notarse que este principio de análisis no compromete demasiado las conclusiones que se obtengan: dado el acuerdo general en la disciplina respecto al carácter recitativo de la poesía hexamétrica, es dable pensar que no había en ella una composición melódica especial. Aunque esto no impide que en ciertos casos, quizás en forma extemporánea, los cantantes ejecutaran un verso en un tono ligeramente diferente al que los acentos de las palabras sugerirían, parece razonable asumir que esto no sucedía de manera regular.

\section{Presentación del texto}

Provista la extensión disponible para este trabajo, me concentraré en él en dos ubicaciones clave del hexámetro: el tercer y el sexto pie. Aunque un análisis de la distribución de acentos así restringido dejará necesariamente dudas sobre el alcance de las conclusiones a las que se llegará, no es plausible en este punto realizar un estudio más amplio. Por lo demás, como se verá, las hipótesis que irán surgiendo a lo largo del trabajo sentarán las bases para uno más detenido sobre cada Idilio o conjunto de Idilios, o eventualmente para una continuación del presente que considere otros datos además de los que se presentarán aquí. En todo caso, el material para concretar ambos proyectos está disponible online.

\footnotetext{
${ }^{15}$ Sobre la cual, cf. Comotti 1989.
} 


\section{Distribución DE ACENTOS}

\section{Tipos de acento en el tercer longum}

La primera ubicación que se estudiará es el longum del tercer pie, en la ubicación de la cesura central del hexámetro. Además de la importancia de esta sílaba, existe evidencia de que a lo largo de la historia del metro fue cambiando la manera en que se la reforzaba acentualmente:

Tabla I.1. Tipos de acento en el tercer longum del hexámetro por autor. Se muestra el porcentaje por fila entre paréntesis ${ }^{16}$.

\begin{tabular}{|l|c|c|c|c|c|}
\hline & Declive & Circunflejo & Ag. en pen. cerr. & Otros ag. en longum & Total \\
\hline Homero & $\begin{array}{c}1180 \\
(62,14 \%)\end{array}$ & $\begin{array}{c}412 \\
(21,70 \%)\end{array}$ & $\begin{array}{c}126 \\
(6,64 \%)\end{array}$ & $\begin{array}{c}181 \\
(9,53 \%)\end{array}$ & 1899 \\
\hline Calímaco & $\begin{array}{c}538 \\
(69,42 \%)\end{array}$ & $\begin{array}{c}138 \\
(17,81 \%)\end{array}$ & $\begin{array}{c}36 \\
(4,65 \%)\end{array}$ & $\begin{array}{c}63 \\
(8,13 \%)\end{array}$ & 775 \\
\hline Nono & $\begin{array}{c}701 \\
(80,57 \%)\end{array}$ & $\begin{array}{c}25 \\
(14,02 \%)\end{array}$ & $(2,87 \%)$ & $\begin{array}{c}22 \\
(2,53 \%)\end{array}$ & 870 \\
\hline
\end{tabular}

Como puede verse, en esta sucesión de los tres autores representativos del desarrollo del hexámetro, el declive barítono es utilizado con mayor frecuencia en detrimento de todos los otros tipos de acento ${ }^{17}$. Si se admite el principio de que el declive es el tipo de acento más demarcativo (porque combina prominencia acentual con descenso del tono, que es en sí mismo un indicador de final de palabra o de frase $)^{18}$, lo que se observa a lo largo de la tradición es una tendencia a marcar el final del primer colon cada vez más enfáticamente ${ }^{19}$. Los

16 Los datos han sido obtenidos a partir de la información disponible en https://greekmps. wordpress.com. «Homero», «Calímaco»y «Nono» corresponden a las muestras de estos autores que han sido publicadas allí. Aquí y en la tabla I.2, no he distinguido entre acentos ante pentemímera y acentos ante cesura trocaica; se requerirá, por lo tanto, un trabajo futuro con un análisis que explore si hay diferentes tendencias en cada caso.

17 La observación no es nueva (cf. sobre esto Maas 1962, §21), pero adquiere, en un análisis métrico-prosódico, un valor por completo diferente al que se le ha dado tradicionalmente en metricología. No ahondaré en este trabajo sobre el problema del acento en Nono, la presentación de cuyos valores es sólo ilustrativa.

18 Cf. Devine y Stephens 1994, pp. 429-431.

19 El cambio no se limita al tipo de acentuación relativo sino que abarca también las proporciones de las cantidades absolutas: los 1180 declives de Homero en el tercer longum 
diferentes Idilios analizados muestran diferencias considerables entre sí en este aspecto:

Tabla I.2. Tipos de acento en el tercer longum del hexámetro de Teócrito por Idilio. Se muestra el porcentaje por fila entre paréntesis.

\begin{tabular}{|c|c|c|c|c|c|}
\hline & Declive & Circunflejo & Ag. en pen. cerr. & Otros agudos & Total \\
\hline Id. I & $68(57,14 \%)$ & $33(27,73 \%)$ & $4(3,36 \%)$ & $14(11,76 \%)$ & 119 \\
\hline Id. II & $82(67,21 \%)$ & $28(22,95 \%)$ & $7(5,74 \%)$ & $5(4,10 \%)$ & 122 \\
\hline Id. III & $19(52,78 \%)$ & $10(27,78 \%)$ & $2(5,56 \%)$ & $5(13,89 \%)$ & 36 \\
\hline Id. IV & $34(65,38 \%)$ & $10(19,23 \%)$ & $5(9,62 \%)$ & $3(5,77 \%)$ & 52 \\
\hline Id. $\mathrm{V}$ & $80(65,04 \%)$ & $33(26,83 \%)$ & $4(3,25 \%)$ & $6(4,88 \%)$ & 123 \\
\hline Id. VI & $19(54,29 \%)$ & $9(25,71 \%)$ & $3(8,57 \%)$ & $4(11,43 \%)$ & 35 \\
\hline Id. VII & $96(72,73 \%)$ & $29(21,97 \%)$ & $2(1,52 \%)$ & $5(3,79 \%)$ & 132 \\
\hline Id. $\mathrm{X}$ & $29(64,44 \%)$ & $13(28,89 \%)$ & $1(2,22 \%)$ & $2(4,44 \%)$ & 45 \\
\hline Id. XI & $42(67,74 \%)$ & $12(19,35 \%)$ & $3(4,84 \%)$ & $5(8,06 \%)$ & 62 \\
\hline Id. XII & $20(64,52 \%)$ & $6(19,35 \%)$ & $2(6,45 \%)$ & $3(9,68 \%)$ & 31 \\
\hline Id. XIII & $39(76,47 \%)$ & $11(21,57 \%)$ & $0(0,00 \%)$ & $1(1,96 \%)$ & 51 \\
\hline Id. XIV & $29(52,73 \%)$ & $16(29,09 \%)$ & $6(10,91 \%)$ & $4(7,27 \%)$ & 55 \\
\hline Id. XVa & $54(61,36 \%)$ & $26(29,55 \%)$ & $3(3,41 \%)$ & $5(5,68 \%)$ & 88 \\
\hline Id. $\mathrm{XVb}$ & $26(70,27 \%)$ & $8(21,62 \%)$ & $1(2,70 \%)$ & $2(5,41 \%)$ & 37 \\
\hline Id. XVI & $54(62,07 \%)$ & $19(21,84 \%)$ & $5(5,75 \%)$ & $9(10,34 \%)$ & 87 \\
\hline Id. XVII & $80(65,57 \%)$ & $25(20,49 \%)$ & $6(4,92 \%)$ & $11(9,02 \%)$ & 122 \\
\hline Id. XVIII & $36(75,00 \%)$ & $6(12,50 \%)$ & $3(6,25 \%)$ & $3(6,25 \%)$ & 48 \\
\hline Id. XXII & $120(63,83 \%)$ & $32(17,02 \%)$ & $19(10,11 \%)$ & $17(9,04 \%)$ & 188 \\
\hline Id. XXIV & $81(71,05 \%)$ & $16(14,04 \%)$ & $10(8,77 \%)$ & $7(6,14 \%)$ & 114 \\
\hline Total Teócrito & $1008(65,16 \%)$ & $342(22,11 \%)$ & $86(5,56 \%)$ & $111(7,18 \%)$ & 1547 \\
\hline
\end{tabular}

representan un 47,5\% del total de versos de su muestra, los 538 de Calímaco un 57,54\% y los 701 de Nono un notable $79,47 \%$. 
La fluctuación, como puede verse, es significativa, pero ciertas tendencias destacan. Los números de los Id. XVIII y XXIV se aproximan a los de Calímaco y, de hecho, se muestran más lejos de Homero incluso que este autor (aunque la diferencia se morigera si se añaden los números de la tercera columna a los de los circunflejos). Esto contradice en cierta medida las observaciones de Fantuzzi, pp. 238-244, que afirma que los poemas bucólicos (v. n. 7) son los más calimaqueos. Que el Id. XXIV muestre una tendencia «moderna» no sorprende: ya Brioso Sánchez (passim) había señalado que es en la "épica» donde Teócrito se muestra más cercano a Calímaco ${ }^{20}$. Pero el $I d$. XVIII pertenece a un grupo que en general se considera más conservador que el resto. Nótese que en este punto la contradicción se exacerba particularmente: no sólo este Idilio es más cercano a Calímaco que a Homero, sino que es el que más se aleja de Homero de todos.

Puede verse también en la tabla que los poemas bucólicos no muestran uniformidad entre sí. Existe, sin embargo, un punto en donde la mayoría de ellos se vincula: un uso más frecuente de circunflejos que Homero y Calímaco y uno menor de declives. Esto es claro en los Id. I, III, VI (tres poemas que además comparten un porcentaje alto de agudos en la cuarta columna) y, aunque no lo he hallado clasificado como bucólico, el Id. XIV (que no comparte la preferencia por agudos, sino más bien lo contrario). Su aparición en este grupo no debería sorprender: aunque no tiene ambiente bucólico, comparte con otros poemas de la colección que sí lo tienen la temática de las quejas de amor y los problemas de los enamorados ${ }^{21}$. Es interesante notar que en los diez versos finales de este texto, que constituyen un pequeño encomio a Ptolomeo, hay siete declives y tan sólo dos circunflejos, es decir, porcentajes más próximos a los del Id. XVb o el VII (ver abajo).

Otros Idilios muestran lo que podría llamarse un criterio «mixto». En algunos aumenta el porcentaje de declives con respecto al modelo homérico (como en los $I d$. XVIII y XXIV), pero no disminuye el de circunflejos, sino que la diferencia está en la caída del número de agudos. Esto es válido para

${ }^{20}$ La contradicción no fue ignorada por Fantuzzi, que trata de salvarla en las pp. 252-253 de su artículo.

${ }^{21}$ Sobre la clasificación genérica del Id. XIV (y X), cf. Cairns 1970. 
los $I d$. VII, XIII y, en menor medida, $\mathrm{II}^{22}$. Otro grupo (el de los $I d$. V y X) parece presentar el fenómeno inverso: aunque tienen un aumento en el porcentaje de circunflejos, no disminuyen el de declives; de nuevo, la diferencia está en la fluctuación en la cantidad de agudos.

Los datos que ofrece el $I d$. XV son particularmente interesantes: mientras que en la parte dialógica (Id. XVa) el estilo es similar al del Id. I y otros, con un alto porcentaje de circunflejos y un nivel más bajo que el homérico de declives (aunque sin el aumento en el número de agudos), en el canto a Adonis (Id. XVb) los porcentajes se modifican notablemente, aproximándose a los del Id. VII. La diferencia es sutil pero significativa y puede haber contribuido a crear un tono diferente para esta sección del poema. Recuerda también que las exigencias del estudio cuantitativo, que hacen muy difícil subdividir cada texto para su estudio detallado, pueden estar haciendo que se pierdan variaciones internas en el tono que podrían ser importantes para el poeta $^{23}$.

Del resto de los poemas, los Id. XII, XVII y XXII son a grandes rasgos homéricos o están entre los números de Homero y Calímaco, y el XI se aproxima mucho a los datos de este último. El Id. IV es quizás el más peculiar de la tabla, pero la diferencia con el resto de los poemas bucólicos se morigera algo cuando se considera el alto porcentaje en él de formas con agudo en sílaba cerrada. Si se cuentan éstas con los circunflejos, el estilo de este texto es similar al del $I d$. V.

Los datos observados en esta sección pueden ser rechazados como accidentales por aquellos que consideren que la no adecuación de muchas de las asociaciones descubiertas a los grupos tradicionales sugiere que son puramente aleatorios. Sin embargo, como ya se ha hecho notar, dentro de esos grupos hay diferencias más que significativas de tono, y es dable imaginar que es precisamente en el tono donde el poeta manifestaría estas distinciones. Por lo demás, no hay un apartamiento completo de los datos presentados de las apreciaciones estándar sobre la colección: los Id. I, III y VI muestran una

${ }^{22}$ No parece haber ningún tipo de criterio que unifique estos tres poemas. Resulta particularmente extraña la distribución acentual del $I d$. VII, en general considerado uno de los dos «programas» poéticos de la bucólica. Como se verá más abajo, su distanciamiento del resto de los Idilios del grupo bucólico no se mantiene en otros análisis.

${ }^{23}$ Pretagostini 1980 ha propuesto que la estructura del tipo A-B-A o A-B-A-B-A es fundamental en el corpus transmitido de Teócrito, lo que refuerza la idea de que sería necesario un estudio que analizara las variaciones formales en el interior de cada Idilio. 
vinculación entre sí y una menor con los $I d$. IV, $\mathrm{V}$ y X, mientras que los $I d$. XVI, XVII y XXII se asocian en su proximidad a los números homéricos. Es importante notar, más allá de estas consideraciones, que la acentuación del tercer longum no puede constituir por sí misma un marcador suficiente para el estilo de un Idilio, por lo que el estudio debe ser complementado con el análisis de otras ubicaciones.

\section{El sexto pie}

Otro punto cardinal del hexámetro es el sexto pie. En este caso, tanto el longum como el biceps resultan importantes para un análisis métrico-prosódico, en la medida en que el último acento del verso puede estar en cualquiera de los dos. La diferencia está en la forma de la palabra final: si es trocaica, el acento estará en el longum, si es espondaica, estará en el biceps ${ }^{24}$. A fin de no multiplicar las tablas, a continuación analizo el tipo de acento final del verso, independientemente de su ubicación efectiva. En otras palabras, los acentos considerados en la tabla I.3 pueden estar tanto en el longum como en el biceps $^{25}$, pero son siempre el último de la línea ${ }^{26}$.

${ }^{24}$ En este punto existe el problema del principio breuis in longo. Sigo aquí la interpretación ofrecida en Abritta 2016, pp. 64-67.

${ }^{25}$ Los datos desagregados por ubicación pueden hallarse en las tablas publicadas on-line para acompañar este artículo.

${ }^{26}$ Por cuestiones del sistema de análisis, la tabla no cuenta versos con monosílabo final no acentuado (sí los que tienen marca de agudo o circunflejo). De cualquier manera, sólo hay 23 en todo el corpus, y en ningún poema hay más de tres casos (el promedio de hecho es 1,28 monosílabos finales no acentuados por Idilio). Dentro del grupo de versos con monosílabo final incluidos, hay 10 versos que tienen acento tanto en biceps como en longum (Id. XI 74, XIII 22, XIII 62, XIII 67, XIV 21, XV 36, XVI 107, XVII 84, XXII 30 y XXII 112). El fenómeno se da 16 veces en la muestra de Homero (una vez cada 155 versos) y sólo una en los Himnos de Calímaco (lo que podría implicar una evasión deliberada del doble acento final en este poeta). La distribución en Teócrito tiene dos peculiaridades: primero, que no hay doble acento en el sexto pie en ninguno de los Idilios bucólicos (exceptuando al XI, si se prefiere incluirlo en este grupo). Segundo, que tanto en el $I d$. XIII como, en menor medida, el XXII hay más casos de doble acento de los que parecería poder esperarse por azar. En el Id. XIII en particular la frecuencia es más de seis veces mayor que en Homero y más de treinta y cinco veces mayor que en Calímaco. En las tablas publicadas online pueden hallarse más detalles sobre este grupo, cuyo estudio detallado no puedo realizar aquí. 
Tabla I.3. Tipo de acento final en el hexámetro de Teócrito por Idilio, en Homero, Calímaco y Nono. Se incluyen palabras trocaicas, espondaicas y monosílabos acentuados. La columna «Circ. Final» incluye, además de los circunflejos, las formas trocaicas paroxítonas con penúltima cerrada por resonante (cf. sec. I.4). Se muestra el porcentaje por fila entre paréntesis.

\begin{tabular}{|c|c|c|c|c|}
\hline & Decl. final & Circ. final & Ag. final & Total \\
\hline Id. I & $82(60,74 \%)$ & $37(27,41 \%)$ & $16(11,85 \%)$ & 135 \\
\hline Id. II & $83(57,64 \%)$ & $42(29,17 \%)$ & $19(13,19 \%)$ & 144 \\
\hline Id. III & $35(66,04 \%)$ & $13(24,53 \%)$ & $5(9,43 \%)$ & 53 \\
\hline Id. IV & $49(77,78 \%)$ & $10(15,87 \%)$ & $4(6,35 \%)$ & 63 \\
\hline$I d . \mathrm{V}$ & $103(68,67 \%)$ & $30(20,00 \%)$ & $17(11,33 \%)$ & 150 \\
\hline Id. VI & $40(86,96 \%)$ & $6(13,04 \%)$ & $0(0,00 \%)$ & 46 \\
\hline Id. VII & $100(64,52 \%)$ & $38(24,52 \%)$ & $17(10,97 \%)$ & 155 \\
\hline Id. $\mathrm{X}$ & $33(57,89 \%)$ & $18(31,58 \%)$ & $6(10,53 \%)$ & 57 \\
\hline Id. XI & $46(60,53 \%)$ & $22(28,95 \%)$ & $8(10,53 \%)$ & 76 \\
\hline Id. XII & $20(55,56 \%)$ & $9(25,00 \%)$ & $7(19,44 \%)$ & 36 \\
\hline Id. XIII & $40(54,79 \%)$ & $16(21,92 \%)$ & $17(23,29 \%)$ & 73 \\
\hline Id. XIV & $35(50,72 \%)$ & $18(26,09 \%)$ & $16(23,19 \%)$ & 69 \\
\hline Id. XVa & $55(54,46 \%)$ & $29(28,71 \%)$ & $17(16,83 \%)$ & 101 \\
\hline Id. $\mathrm{XVb}$ & $31(68,89 \%)$ & $7(15,56 \%)$ & $7(15,56 \%)$ & 45 \\
\hline Id. XVI & $69(63,30 \%)$ & $24(22,02 \%)$ & $16(14,68 \%)$ & 109 \\
\hline Id. XVII & $74(55,22 \%)$ & $43(32,09 \%)$ & $17(12,69 \%)$ & 134 \\
\hline Id. XVIII & $26(48,15 \%)$ & $15(27,78 \%)$ & $13(24,07 \%)$ & 54 \\
\hline Id. XXII & $123(55,91 \%)$ & $61(27,73 \%)$ & $36(16,36 \%)$ & 220 \\
\hline Id. XXIV & $78(56,12 \%)$ & $44(31,65 \%)$ & $17(12,23 \%)$ & 139 \\
\hline Total Teócrito & $1122(60,36 \%)$ & $482(25,93 \%)$ & $255(13,72 \%)$ & 1859 \\
\hline Homero & $1516(63,78 \%)$ & $523(22 \%)$ & $338(14,22 \%)$ & 2377 \\
\hline Calímaco & $575(63,12 \%)$ & $189(20,75 \%)$ & $147(16,14 \%)$ & 911 \\
\hline Nono & $563(67,83 \%)$ & $188(22,65 \%)$ & $79(9,52 \%)$ & 830 \\
\hline
\end{tabular}


Nuevamente, la fluctuación es considerable, pero en este caso algunos aspectos de la distribución parecen más marcados que en la tabla I.3. Nótese en particular que los Idilios bucólicos muestran en general (la excepción es el Id. I, que es el que más se aproxima al sexto pie de los no-bucólicos - ver infra -; cf. sin embargo la sec. II.3) un porcentaje de declive final mayor que Homero y Calímaco y (junto con el $I d$. XI) que el resto de los poemas ${ }^{27}$. Este grupo también se destaca por un uso mucho menos frecuente de agudos finales (un promedio de $8,64 \%$ frente a uno de $16,84 \%$ del resto), lo que sugiere una cierta tendencia a buscar un final de verso con tono descendente.

Destaca en el grupo el $I d$. VI, si se considera que es uno de los que mayor porcentaje de circunflejos en el tercer pie tiene. En este breve poema se observa una combinación frecuente de acentos en los dos pies que cierran cada parte del hexámetro en un orden tonal descendente, es decir, un primer colon terminando en un tono más alto que el segundo ${ }^{28}$. Es un fenómeno sobre el que se volverá más abajo.

Los Idilios XVb y XVI comparten con los recién analizados el uso más frecuente de declives, pero no muestran uno demasiado reducido de agudos, sino más bien una baja considerable en el de circunflejos (frente al resto de la colección). En el Id. XVI la explicación podría tener que ver con la época de composición ${ }^{29}$ : quizás Teócrito había definido el estilo de la bucólica en este periodo y lo respeta mayormente en este texto, con la salvedad de que hace un uso más regular de agudos para individualizar el tono del encomio

${ }^{27}$ Una revisión rápida de las muestras que pueden hallarse en https://greekmps.wordpress. com indica que el autor con el porcentaje más alto de declives finales es Opiano de Cilicia, con $71 \%$ de sus versos terminando con este tipo de acento (sobre otros aspectos de la métrica de este autor, cf. Silva Sánchez 1993). Sólo se le aproxima Apolonio, que tiene 66\%. En semejante contexto, el $78 \%$ del $I d$. IV y sobre todo el $87 \%$ del $I d$. VI son espectacularmente peculiares.

${ }^{28}$ Recuérdese (sec. I.4) con respecto a esto que el circunflejo era considerado por los antiguos ỏ $v ́ \beta \alpha \rho v \varsigma$, lo que implica naturalmente que tendría un sonido más agudo que el declive.

${ }^{29}$ Este complejísimo problema ha sido motivo de mucha discusión. Cf. por ejemplo la presentación de Gow 1952, pp. xvii-xix. En el caso del Id. XVI puede asumirse con relativa seguridad una datación temprana, dada la temática del texto (difícilmente un Teócrito ya establecido en Alejandría escribiría un encomio a Hierón de Siracusa para solicitar su patronazgo). En todo caso, la explicación que se ofrece no debe entenderse más que como especulativa. Otro rasgo excepcional del $I d$. XVI es el porcentaje de espondeos (30\%), sobre lo cual cf. Brioso Sánchez, p. 67, n. 93. Fantuzzi, p. 252, sospecha también que esta peculiaridad es producto de la época de composición del poema. 
(además de las diferencias en el tercer pie, donde el $I d$. XVI sigue el estilo homérico). En el caso del $I d$. XVb el aumento en el uso de declives puede tener un doble objetivo: diferenciarse de la parte dialógica del poema (con un porcentaje alto de circunflejos en el final del verso como en el final del primer colon; cf. sec. II.1) y darle al canto de Adonis un tono que podría ser interpretado como más grandilocuente.

Los Id. XIV y XVIII, destacan dentro de la colección por el bajo porcentaje de declives finales y, sobre todo, el alto de agudos ${ }^{30}$. En el caso del primero, el dato contrasta con lo observado en la sec. II.1 sobre su afinidad con el tono de los Id. I, III y VI; quizás la diferencia sirve para dar al poema un sonido distinto a estos. Por lo demás, el uso frecuente de circunflejos y agudos en sílaba cerrada en el tercer pie (40\% de los versos del poema entre los dos tipos), combinado con un uso frecuente de circunflejos y agudos finales daría al texto una suerte de melodía sostenida en tono alto. Esto mismo pero en forma mucho más marcada se observa en el $I d$. XVIII. La regularidad del tercer pie con declive barítono y el alto porcentaje de agudos finales implica un tono siempre ascendente para los versos de este epitalamio, coherentes con un poema que tiene carácter celebratorio ${ }^{31}$.

El resto de los poemas de la colección muestran lo que podría denominarse un estilo propio de Teócrito: un porcentaje de declives más bajo que el de Calímaco y Homero, un uso más frecuente de circunflejos y uno de agudos

${ }^{30}$ También el $I d$. XIII, pero es otro caso extraño en la medida en que tiene un alto porcentaje de agudos pero uno de declives similar al de Calímaco. Es interesante notar que casi un tercio de los agudos finales (5 de 17) se concentran entre los versos 53-62 (el v. 61 parece ser una interpolación), en la búsqueda desesperada de Hilas por Heracles. Es otro buen ejemplo de que dentro de cada poema pueden utilizarse los acentos para construir un cierto tono.

${ }^{31}$ No debe verse una contradicción entre esta observación y lo señalado en la n. 32 sobre la acumulación de agudos en el pasaje de la búsqueda de Hilas en el Id. XIII. En la performan$c e$ efectiva del texto el tono acentual sería secundado por la interpretación del recitador, que diferenciaría entre tonos agudos propios de una celebración y propios de un lamento. Aunque esto es sólo especulación, no es una que parezca particularmente arriesgada. Difícilmente

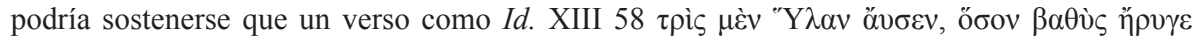
$\lambda \alpha \mu$ ó «Tres veces llamó a Hilas, tan fuerte como gritaba su garganta» sería recitado con

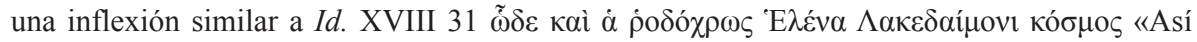
también es Helena de rosada piel adorno para Lacedemonia». Ambas líneas tienen un declive barítono central seguido de agudos, pero en la primera cualquier buen cantante enfatizaría la desesperación del grito de Heracles y en la segunda la alegría de las mujeres por el casamiento de su compañera. 
en que fluctúa entre el $10 \%$ y el $20 \%$. Algunos poemas se aproximan al estilo bucólico (como el Id. X, el XVII o el XXIV, donde la diferencia está en el porcentaje de declives), otros (como el $I d$. XII o el XVa) al de los Id. XIII y $\mathrm{XIV}$, pero en general no hay textos que se destaquen especialmente en este grupo. Como rasgo general, parece plausible afirmar que Teócrito prefería una mayor variación en su poesía no-bucólica entre versos de tono ascendente y versos de tono descendente, si el alto porcentaje de circunflejos en el sexto pie puede ser tomado como indicio.

El análisis del sexto pie sugiere nuevamente que el corpus de Idilios exhibía una diversidad notable de estrategias, pero refuerza la idea de que dentro de él hay ciertos usos que se repiten. En este caso, los Idilios bucólicos muestran una mayor uniformidad que en el estudio del tercer pie. Los Id. XIII, XIV y XVIII se destacan también por el alto porcentaje de agudos finales. Una vez más, la diferenciación de las dos partes del $I d$. XV sugiere una adecuación del tono al tema o, en otras palabras, una utilización del aspecto métrico-prosódico de la composición para dar, a falta de una mejor expresión, una cierta coherencia auditiva a los diferentes poemas y a las diferentes partes de cada poema.

\section{Algunas correlaciones acentuales}

Los datos analizados hasta ahora sugieren que en algunos Idilios había una tendencia a dar a los versos un tono ascendente desde el comienzo al final $\mathrm{y}$ en otros un tono descendente ${ }^{32}$. Si esto era efectivamente así, es dable pensar que tendría consecuencias que trascienden la simple proporción de acentos en las diferentes ubicaciones del verso: algunas combinaciones se favorecerían para generar la percepción del ascenso o descenso de la melodía, mientras que otras se evitarían para prevenir lo contrario. En Idilios como

${ }^{32}$ Esta consideración debe realizarse dejando de lado el problema de la catátesis (cf. Devine y Stephens 1994, pp. 435-455, esp. 441-445, y Connell 2011). Siendo este un efecto lingüístico automático, no es implausible que los oyentes percibieran un verso como Id. XIII

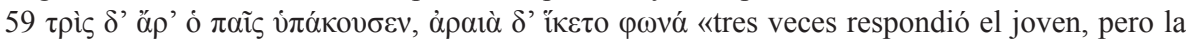

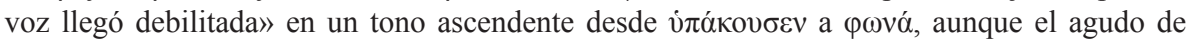
la segunda palabra se pronunciara en una frecuencia real más baja que el de la última sílaba de la primera. Esto es, por supuesto, asumiendo que el ejecutor no compensara en su lectura el fenómeno de la catátesis para mantener el efecto (lo que los textos con notación musical sugieren que no sucedía). 
los bucólicos, donde se ha observado una inclinación a buscar un tono bajo

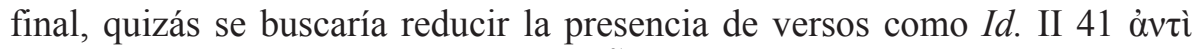

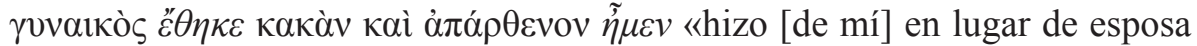
una mujer desdichada y no más una doncella», donde el declive central y el circunflejo final darían a la línea un sonido que podría ser percibido como un ascenso. A la inversa, en un Idilio como el XVIII, donde se ha observado una preferencia por finales en tono alto, quizás se evitarían versos como Id.

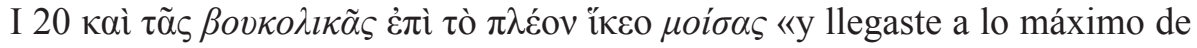
la poesía pastoril», donde después del circunflejo central se concluye con un declive en la última sílaba.

Para verificar si de hecho este tipo de fenómenos se observan en el texto conservado, he realizado una serie de análisis sobre cada poema que pueden hallarse en los documentos publicados online. La cantidad de tablas y datos implicados impide reducir los resultados al tamaño necesario para introducirlos en el espacio de este artículo. Sin embargo, dado que los números están disponibles para su consulta, no hay motivos para no estudiarlos aquí.

Una prueba fundamental para analizar si hay algún tipo de correlación acentual en un Idilio es la verificación del valor $\Omega$ entre dos variables: presencia de circunflejo o declive en el tercer pie y presencia de circunflejo o declive en el sexto $^{33}$. A través de una serie de pasos intermedios, esto permite llegar a una tabla como la siguiente, para la que utilizo el Id. I como ejemplo:

Tabla I.4. Correlación entre tipo de acento en el tercer longum y tipo de acento en el sexto pie en el Idilio I de Teócrito. Se incluye el porcentaje del total de versos analizados en la tabla entre paréntesis.

\begin{tabular}{|l|c|c|c|}
\hline & Circunflejo en sexto pie & Declive en sexto pie & Total \\
\hline Circunflejo en tercer longum & $14(15,91 \%)$ & $14(15,91 \%)$ & 28 \\
\hline Declive en tercer longum & $16(18,18 \%)$ & $44(50 \%)$ & 60 \\
\hline Total & 30 & 58 & 88 \\
\hline
\end{tabular}

Cada celda muestra una combinación posible de acento en el tercer pie y acento en el sexto pie; la primera, por ejemplo, muestra que hay 14 versos en

${ }^{33}$ Nótese que estas condiciones sólo incluyen en el estudio versos que tengan acentos de este tipo tanto en el tercer como el sexto pie, que representan un porcentaje del total que fluctúa de $46 \%$ a $81 \%$ (con un promedio de $60 \%$ ). Esto implica que será necesario corroborar este análisis con pruebas que incluyan al resto de las líneas. 
el Id. I con un circunflejo en el tercer longum y un circunflejo en el sexto pie $^{34}$. Hay cuatro valores $\Omega$ posibles para esta tabla (dependiendo qué conjunto de variables se combinen; el número concreto del valor varía pero su interpretación no), pero el que se usará aquí es el que estima la diferencia en la posibilidad de la combinación circunflejo-circunflejo frente a circunflejodeclive sobre la posibilidad de la combinación declive-circunflejo frente a declive-declive. En otras palabras, cuánto más posible es hallar la combinación circunflejo-circunflejo respecto a la combinación circunflejo-declive de lo que es hallar la combinación declive-circunflejo respecto a la combinación declive-declive. Para esta tabla, ese valor es 2,75. Para llegar a ese número se estima la razón de circunflejos en el tercer longum entre los dos tipos de acento en el sexto pie, con la segunda columna como divisor (14/14=1) y lo mismo para los declives en el tercer longum $(16 / 44=0,36 \ldots$ o algo más de un declive en el tercer longum con circunflejo en el sexto pie por cada tres declives en el tercer longum con declive en el sexto pie). El primer número se divide por el segundo, lo que da la razón de razones (el valor $\Omega$ ), que en este caso es $1 / 0,36 \ldots=2,75^{35}$.

Este resultado implica que es bastante más probable $(2,75$ veces más

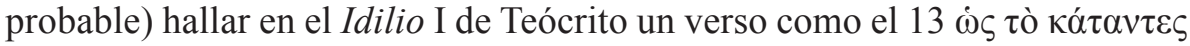

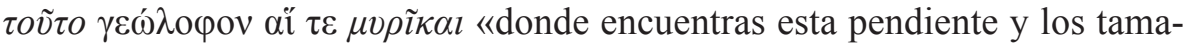

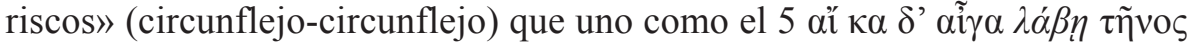
$\gamma \dot{\varepsilon} \rho \alpha \varsigma, \grave{\varepsilon} \varsigma \tau \dot{\varepsilon} \kappa \alpha \tau \alpha \rho \rho \varepsilon \tilde{\imath}$ «si aquel toma la cabra como premio, [la chiva] te caerá [a ti]» (declive-circunflejo), lo que puede resultar extraño si se toma en cuenta que hay 14 del primer tipo y 16 del segundo, pero es perfectamente coherente si se considera la distribución relativa con versos como el 20 (declive-circunflejo; citado más arriba, al principio de esta sección) y el 1

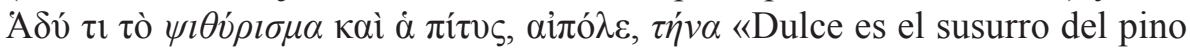
aquel, cabrero» ${ }^{36}$ (declive-declive). En otras palabras, dada una cierta cantidad de versos con declive y circunflejo en el tercer pie, el poeta evita la combinación declive-circunflejo o, si se quiere, favorece la combinación

${ }^{34}$ A partir de este punto, para simplificar la exposición, utilizaré la nomenclatura «circunflejo-circunflejo» para esta combinación y el mismo modelo para el resto («declive-circunflejo», por ejemplo, aludirá a los versos con un declive barítono en el tercer longum y un circunflejo en el sexto pie).

${ }^{35}$ Sobre la utilidad de este valor para el estudio de fenómenos literarios, cf. Kenny 1982, pp. 68-70. Una explicación más detallada puede hallarse en Agresti 2007, pp. 28-32.

${ }^{36}$ Para la traducción de este verso repongo el sentido que se completa con el siguiente. 
circunflejo-circunflejo. Esto corrobora la hipótesis ofrecida arriba de que en la bucólica hay una búsqueda de un cierto tono descendente.

El fenómeno se repite de manera clara en los Id. III $(\Omega=6)$, XI $(\Omega=3,67)$ y XVI $(\Omega=9,07)$, con una salvedad: mientras que la distribución de porcentajes del total en los $I d$. III y XI es similar a la del $I d$. I, en el XVI se observa un uso mucho más frecuente de la combinación circunflejo-circunflejo ( $36 \%$ de los casos, contra un $12 \%$ del III y $8,16 \%$ del XI). En otras palabras, aunque la tendencia a evitar el ascenso del tono persiste, no parece haber una búsqueda tan marcada de un tono descendente. Como se ha notado ya, el $I d$. XVI parece compartir con los Idilios bucólicos diversos rasgos que lo diferencian de otros poemas, pero conservando una cierta individualidad que también los distingue de aquellos. Si la explicación de esto es, como se ha señalado, la época de composición del poema o debe buscarse una motivación más deliberada, es un tema que debe quedar para otros trabajos.

Los valores $\Omega$ del resto de los Idilios bucólicos no parecen favorecer la hipótesis presentada ${ }^{37}$. Sin embargo, un análisis más detallado permite inferir que esto es producto de una cierta variación en la estrategia compositiva en ellos, y no de una diferencia real de estilo. En efecto, todos los poemas del grupo muestran un valor porcentual para la combinación declive-circunflejo (es decir, tono ascendente) más bajo (Id. IV=10,53\%; V=14,14\%; $\mathrm{VI}=7,41 \%$; VII $=16,98 \%$ ) que el de todo el resto de la colección (un promedio de 23,55\%; 24,49\% excluyendo XI; 25,48\% excluyendo también XVI). Nótese que los valores de hecho son marcadamente más bajos en general que el porcentaje en el $I d$. I $(18,18 \%)$, donde el fenómeno se verificó en forma contundente.

Dos poemas se vinculan por sus bajos valores $\Omega$, el $I d$. X $(0,292)$ y el $I d$. XIV $(0,267)^{38}$, que indican que en ellos es casi cuatro veces más probable que se encuentre un declive en el tercer longum combinado con un circunflejo en el sexto pie que dos circunflejos en esas dos ubicaciones (lo inverso a lo observado en el $I d$. I). Del segundo se ha notado en la sección II.2 que parece mostrar una tendencia al mantenimiento del tono, lo que no parece del todo coherente con esta observación. En todo caso, el rasgo más peculiar de estos

${ }^{37}$ Son los siguientes: $\mathrm{IV}=0,78 ; \mathrm{V}=0,52 ; \mathrm{VI}=1,21 ; \mathrm{VII}=1,43$. Aunque los últimos dos son positivos, son relativamente bajos comparados con los otros.

${ }^{38}$ La vinculación entre ambos también ha sido observada por Cairns (1970), que los considera representantes del mismo género. 
textos es su variabilidad acentual: aunque ambos tienen niveles porcentuales bajos de la combinación circunflejo-circunflejo, son los poemas donde menor diferencia hay entre las otras tres. De hecho, el $I d$. XIV tiene los porcentajes más altos de la colección de acentos cruzados $(30,30 \%$ de declive-circunflejo y $27,27 \%$ de circunflejo-declive) y el más bajo de la combinación declivedeclive $(36,36 \%)$. Como puede verse, la tendencia en este texto es a utilizar casi en la misma medida este conjunto de combinaciones. Aunque el $I d$. X tiene un porcentaje más bajo en la combinación circunflejo-declive, es mucho más variado que cualquier otro de la colección (excepto, obviamente, el XIV).

Por último, el valor $\Omega$ del $I d$. XVIII no puede calcularse porque no hay en él ni un solo caso de combinación circunflejo-circunflejo. Sin embargo, los datos de la correlación acentual sugieren que la hipótesis de una tendencia al tono ascendente ofrecida en la sec. II.2 es correcta: sólo $11,76 \%$ de los versos analizados muestra la combinación estrictamente descendente (circunflejo-declive), y el porcentaje de líneas con la combinación ascendente (declive-circunflejo) es el tercero más alto de la colección $(29,41 \%)$.

Puede concluirse este apartado, por lo tanto, indicando que, aunque, como se ha observado en la n. 33, es necesario revisar todos estos resultados con estudios que incluyan la totalidad (o al menos la mayor parte) de los versos de cada poema, las pruebas realizadas hasta aquí corroboran las propuestas realizadas en las secciones anteriores de este artículo.

\section{RESUMEN Y CONCLUSIONES}

Por los motivos señalados al comienzo de este trabajo, es difícil saber en qué medida los análisis realizados serán verificados conforme avance la investigación métrico-prosódica de la poesía griega antigua en general, del hexámetro en particular y especialmente del hexámetro helenístico. A medida que se descubran las reglas (si las había) que regulaban la distribución de los acentos en las distintas formas o ubicaciones de los versos, es posible que ciertos aspectos del metro de Teócrito resulten menos (o más) extraños o peculiares.

Establecido eso, la variedad de estrategias compositivas dentro de la colección de Idilios (reconocida ampliamente por la interpretación literaria, la lingüística y la metricología) sugiere que los resultados obtenidos son realmente producto de determinaciones deliberadas del poeta.

Los poemas bucólicos (I, III-VII), aunque con fluctuaciones internas, sobre todo en la distribución acentual del tercer pie, parecen haberse caracteri- 
zado por un tono descendente desde el final del primer colon al final del segundo. Dos interpretaciones de esto son posibles: que el autor buscaba dar un tono más grave o grandilocuente a este tipo de poemas, quizás un tono más propio de una lamentación (asumiendo que la melodía descendente generara semejante tono), o que la línea descendente fuera una manera de demarcar más claramente un verso de otro, lo que quizás daba un cierto carácter «rural» a los poemas. Si se toma en cuenta que la poesía alejandrina sofisticada hace uso mucho más frecuente del encabalgamiento que el hexámetro tradicional arcaico, es plausible que la demarcación de cada línea con un tono descendente contribuyera a generar un sonido alejado de la sofisticación contemporánea a Teócrito.

El Id. XI, que es considerado bucólico por muchos autores, no ha mostrado en el estudio métrico-acentual la peculiaridad que su métrica interna exhibe. Aunque, como otros, tiene un tercer pie que no responde al estándar que parecería establecer el $I d$. I, muestra la misma correlación entre los finales de cada parte que éste y por lo tanto podría decirse que tiene una distribución acentual «bucólica». Esto no resulta particularmente sorprendente.

Más interesantes son los datos que corresponden al Id. XVI. Se ha notado a lo largo del análisis que este poema comparte rasgos con los bucólicos, pero siempre con algún tipo de desplazamiento que lo diferencia. No puede dejar de verse en esta estrategia compositiva a un poeta que busca distinguir el estilo individual de un texto del de sus otras composiciones. Si esto es porque el $I d$. XVI fue escrito en la misma época que la poesía bucólica o por otros motivos, no puede ser considerado aquí.

El $I d$. XXIV ha demostrado ser el más «moderno» de toda la colección, con una distribución acentual en el tercer pie que por momentos se parece más a la de Nono que a la de Calímaco, por no mencionar a Homero, y un sexto pie propiamente teocríteo. La crítica ha interpretado este poema como un típico tratamiento helenístico de un tema mitológico, y la forma en que Teócrito lo compone contribuye a reforzar la idea de que busca alejarse lo más posible de las formas tradicionales.

El Id. XVIII muestra una estrategia diferente para diferenciarse del resto de la colección y quizás de la tradición que los precede. El uso frecuente de agudos finales combinado con una cantidad notable de declives barítonos en el tercer pie da a esta composición un cierto tono ascendente, que también se observa en el análisis de la correlación acentual. El carácter celebratorio del 
texto parece poder explicar este fenómeno, pero quizás este estilo de melodía ascendente fuera propio del epitalamio.

El $I d$. XIV y el $I d$. X han aparecido asociados recién en el último conjunto de pruebas realizado, que los muestra como poemas que hacen uso de una considerable variabilidad acentual. Aunque la vinculación entre ambos puede sostenerse desde otras perspectivas (cf. Cairns 1970), las diferencias en la distribución de acentos efectiva tanto en el tercer como en el sexto pie sugieren que es necesario ser precavidos y no apresurar conclusiones sobre la base de un único resultado.

El Id. XV ha mostrado en el estudio del tercer y del sexto pie una tendencia a variar la técnica compositiva cuando el contenido del poema cambia. En XVb, el canto a Adonis, tanto en el final del primer colon como en el final del segundo hay un aumento en la proporción de declives barítonos. De hecho, en esa sección no hay un solo ejemplo de combinación circunflejo en el tercer longum y circunflejo en el pie final. Más allá del valor que tendría en sí misma para señalar el cambio entre las secciones, la dirección de la diferencia puede sugerir que el uso más frecuente de declives daba un tono más elevado a la poesía (¿quizás un tono más moderno?). Un estudio más detenido de este texto y quizás de otros en donde haya un giro similar es necesario para corroborar esta hipótesis.

He realizado algunas observaciones aisladas sobre los Id. II, XII, XIII, XVII y XXII. El primero parece mostrar algunos rasgos calimaqueos, pero con peculiaridades propias, como un uso menor de agudos en el tercer pie y un sexto pie teocríteo. El $I d$. XIII ha probado ser un texto con singularidades notables (cf. las n. 26 y 30). Los Id. XII y XVII tienen un tercer pie homérico, pero traicionan las expectativas en el sexto, donde muestran la distribución característica de otros poemas no-bucólicos de la colección (más circunflejos y menos declives). Lo mismo puede decirse del XXII, con su tercer pie calimaqueo pero su alto porcentaje de circunflejos finales.

Como se señaló al comienzo, lo importante de este estudio no eran tanto las interpretaciones de los datos sino su presentación. Más allá de lo mucho que podría decirse a partir de los números que se han expuesto y estudiado y de lo mucho más que podría agregarse con los que no lo han sido, de lo analizado parece poder extraerse con cierta certeza una conclusión: el estudio métrico-acentual de la poesía de Teócrito merece un lugar en la lectura de su poesía tan importante como el que tienen el metricológico y el dialectal. 


\section{BIBLIOGRAFÍA}

Abritta, A. 2015: «On the Role of Accent in Ancient Greek Poetry. Pitch Patterns in the Homeric Hexameter», QUCC 111, pp. 11-27.

Abritta, A. 2016: «Sobre el problema de la paroxitonesis», Habis 47, pp. 63-82.

Agresti, A. 2007: An Introduction to Categorical Data Analysis, Nueva Jersey.

Allen, S. W. 1967: «Correlations of Tone and Stress in Ancient Greek», en To Honor Roman Jakobson, vol. 1, La Haya, pp. 46-62.

Allen, S. W. 1973: Accent and Rhythm, Cambridge.

Brioso Sánchez, M. 1976-77: «Aportaciones al estudio del hexámetro de Teócrito», Habis 7, pp. 21-56 y Habis 8, pp. 57-76.

Cairns, F. 1970: «Theocritus Idyll 10», Hermes 98, pp. 38-44.

Cavallero, P. A. 1998: «Dos propuestas de crítica textual sobre el Idilio II de Teócrito (vv. 61 y 164)», Emerita 66, pp. 307-319.

Comotti, G. 1989: «Melodia e accento di parola nelle testimonianze degli antichi e nei testi con notazione musicale», QUCC 32, pp. 91-108.

Connell, B. 2011: «Downstep», en van Oostendorp, M., Ewen, C. J., Hume, E. y Rice, K. (eds.), The Blackwell Companion to Phonology, West Sussex, pp. 824-847.

David, A. P. 2006: The Dance of the Muses. Choral Theory and Ancient Greek Poetics, Oxford.

Devine, A. M. y Stephens, L. D. 1991: «Dionysius of Halicarnassus, De Compositione Verborum XI: Reconstructing the Phonetics of the Greek Accent», TAPhA 121, pp. 229-286.

Devine, A. M. y Stephens, L. D. 1994: The Prosody of Greek Speech, Nueva York.

Di Benedetto, V. 1956: «Omerismi e struttura metrica negli idilli dorici di Teocrito», A SNP 25, pp. 48-60.

Fantuzzi, M. 1995: «Variazione sull'esametro in Teocrito», en Fantuzzi, M. y Pretagostini, R. (eds.), Struttura e storia dell'esametro greco, vol. I, Roma, pp. 221-264.

Gow, A. S. F. 1952: Theocritus, 2 vols., Cambridge.

Hunt, J. M. 2009: Fiction and Mimesis in the Idylls of Theocritus, Tesis de doctorado, Brown University, Rhode Island, $<$ https://repository.library.brown.edu/studio/ item/bdr\%3A89>.

Hunter, R. 1996: Theocritus and the Archaeology of Greek Poetry, Cambridge.

Kenny, A. 1982: The Computation of Style, Oxford.

Lascoux, E. 2003: «Écouter Homère? Pour un codage tonotopique de l'épos», Gaia 7, pp. 309-319.

Maas, P. 1962: Greek Metre, trad. H. Lloyd-Jones, Oxford.

Nagy, G. 2000: «Reading Greek Poetry Aloud. Evidence from the Bacchylides Papyri», QUCC 64, pp. 7-28. 
Nagy, G. 2010: «Language and Meter», en Bakker, E. J. (ed.), A Companion to the Ancient Greek Language, West Sussex, pp. 370-387.

O’Neill, E. G. (Jr.) 1942: «The Localization of Metrical Word Types in the Greek Hexameter», YClS 8, pp. 103-178.

Pretagostini, R. 1980: «La struttura compositiva dei carmi teocritei», QUCC 5, pp. 57-74.

Probert, P. 2003: A New Short Guide to the Accentuation of Ancient Greek, Londres.

Sicking, C. M. J. 1993: Griechische Verslehre, Múnich.

Silva Sánchez, T. 1993: «Sobre una particularidad del hexámetro de Opiano de Anazarbo», Excerpta Philologica 3, pp. 115-125.

Sturtevant, E. H. 1920: The Pronunciation of Greek and Latin. The Sounds and Accents, Chicago.

West, M. L. 1982: Greek Metre. Oxford.

Fecha de recepción de la primera versión del artículo: 29/11/2016

Fecha de aceptación: 23/01/2017

Fecha de recepción de la versión definitiva: 30/01/2017 